\title{
Production and study of new neutron rich heavy nuclei in multinucleon trans- fer reactions
}

\author{
V.I. Zagrebaev ${ }^{1, a}$ and Walter Greiner ${ }^{2, b}$ \\ ${ }^{1}$ Flerov Laboratory of Nuclear Reactions, JINR, Dubna, Moscow Region, Russia \\ ${ }^{2}$ Frankfurt Institute for Advanced Studies, J.W. Goethe-Universität, Frankfurt, Germany
}

\begin{abstract}
Problems of production and study of new neutron-enriched heavy nuclei are discussed. Lowenergy multinucleon transfer reactions are shown to be quite appropriate for this purpose. Reactions with actinide beams and targets are of special interest for synthesis of new neutron-enriched transfermium nuclei and not-yet-known nuclei with closed neutron shell $N=126$ having the largest impact on the astrophysical r-process. The estimated cross sections for the production of these nuclei look very promising for planning such experiments at currently available accelerators. These experiments, however, are rather expensive and difficult to perform because of low intensities of the massive projectile beams and problems of separating and detecting the heavy reaction products. Thus, realistic predictions of the corresponding cross sections for different projectile-target combinations are definitely required. Some uncertainty still remains in the values of several parameters used for describing the low-energy nuclear dynamics. This uncertainty does not allow one to perform very accurate predictions for the productions of new heavier-than-target (trans-target) nuclei in multinucleon transfer reactions. Nevertheless these predictions are rather promising (large cross sections) to start such experiments at available accelerators if the problem of separation of heavy transfer reaction products would be solved.
\end{abstract}

\section{Motivation}

The upper part of the present-day nuclear map consists mainly of proton rich nuclei, while the unexplored area of heavy neutron enriched nuclides (also those located along the neutron closed shell $N=126$ to the right-hand side of the stability line) is extremely important for nuclear astrophysics investigations and, in particular, for the understanding of the $r$ process of astrophysical nucleogenesis. For elements with $Z>100$ only neutron deficient isotopes (located to the left of the stability line) have been synthesized so far. Due to the bending of the stability line toward the neutron axis, in fusion reactions only proton-rich isotopes of heavy elements can be produced. That is the main reason for the impossibility of reaching the center of the island of stability $(Z \sim 110 \div 120$ and $N \sim 184)$ in the superheavy (SH) mass region by fusion reactions with stable projectiles. Because of that we also have almost no information about neutron-rich isotopes of heavy elements located in the "northeast" part of the nuclear map: for example, there are 19 known neutron-rich isotopes of cesium $(Z=55)$ and only 4 of platinum $(Z=78)$ (see Fig. 1). Thus, the whole "northeast" area of the nuclear map is still terra incognita. Production and studying properties of nuclei located in this region will open a new field of research in nuclear physics.

\footnotetext{
ae-mail: zagrebaev@jinr.ru

be-mail: greiner@fias.uni-frankfurt.de
}

There are only three methods for the production of heavy elements, namely, fusion reactions, a sequence of neutron capture and $\beta^{-}$decay processes and multi-nucleon transfer reactions.

\section{Fusion Reactions}

Fusion reactions of stable projectiles (even with actinide targets) lead to formation of neutron deficient isotopes of heavy elements located to the left of the stability line. Recently, many speculations appeared on the use of radioactive ion beams for the synthesis and study of new heavy elements and isotopes. The use of accelerated neutron enriched fission fragments for the production of SH nuclei in rather symmetric fusion reactions does not look very encouraging and needs beam intensities at the hardly reachable level of $10^{13}$ pps or higher [1]. The lighter radioactive ions could be more useful here. In fusion reactions of ${ }^{18-20} \mathrm{~N},{ }^{20-22} \mathrm{O}$ or ${ }^{22-24} \mathrm{~F}$ with actinide targets new (though not so much neutron enriched) isotopes of elements with $Z>100$ might be synthesized at rather low beam intensities of $10^{8}$ pps owing to much larger cross sections [1].

A hypothetical pathway to the middle of the island of stability was proposed recently owing to possible $\beta^{+}$decay of SH nuclei located from the up-left side of this island [2]. Such nuclei could be formed in ordinary fusion reactions ${ }^{48} \mathrm{Ca}+{ }^{250} \mathrm{Cm}$ and/or ${ }^{48} \mathrm{Ca}+{ }^{249} \mathrm{Bk}$. In these reactions relatively neutron rich isotopes of $\mathrm{SH}$ elements 114 and 


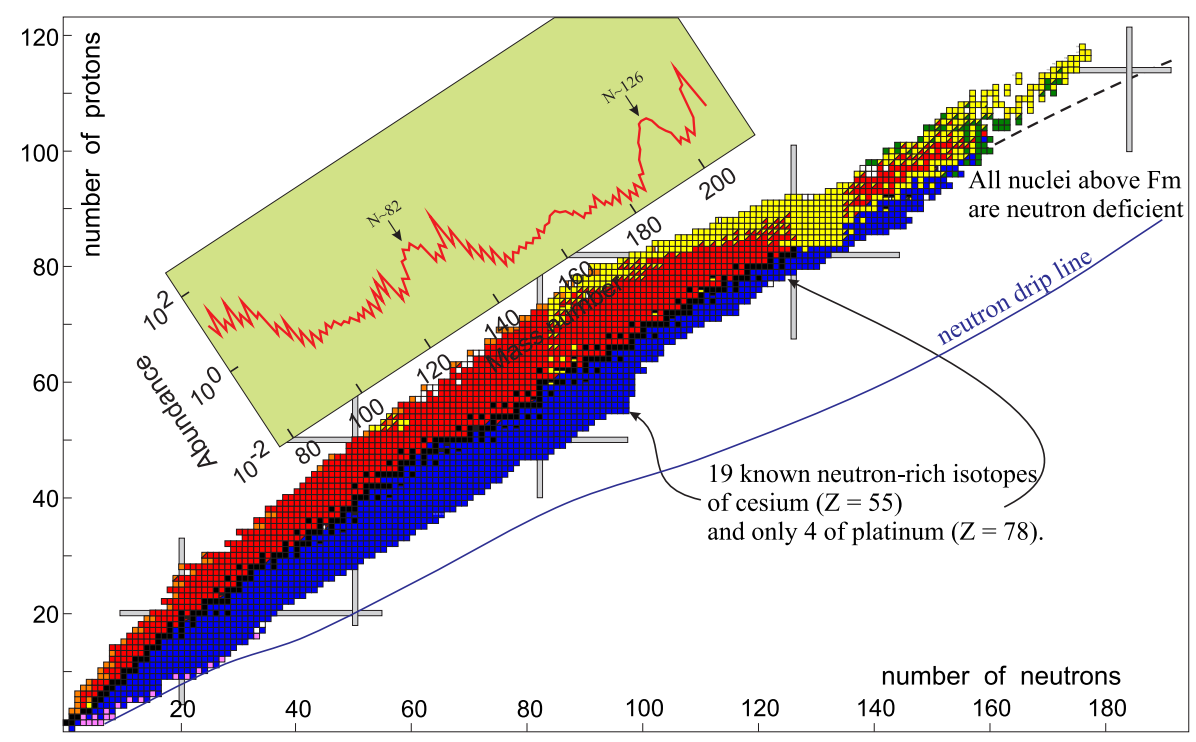

Figure 1. Nuclear map as it looks today. Gray strips indicate positions of proton and neutron closed shells. On the inset relative abundance of the elements in the universe is shown with clear visible maxima in the regions of the "waiting points" along the neutron closed shells $N=82$ and $N=126$.

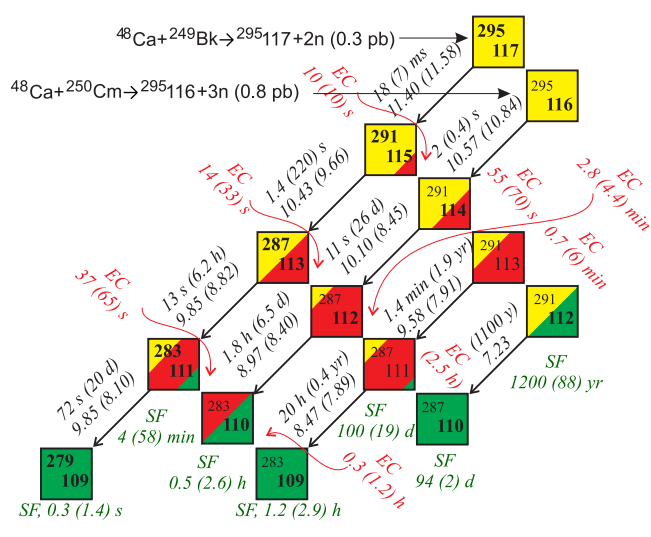

Figure 2. The pathway to the middle of the island of stability via a possible $\beta^{+}$decay of the isotopes ${ }^{291} 115$ and ${ }^{291} 114$.

115 are formed as $\alpha$ decay products of the corresponding evaporation residues. These isotopes should have rather long half-lives and, thus, they could be located already in the "red" area of the nuclear map, i.e., they may be $\beta^{+}$decaying nuclei. In Fig. 2 possible decay chains of these isotopes are shown along with the corresponding values of $Q_{\alpha}$ and half-lives. In accordance with our calculations, the isotopes ${ }^{291} 115$ and ${ }^{291} 114$ may experience not only $\alpha$ decay but also electron capture with half-life of several seconds. If it is really true, the narrow pathway to the middle of the island of stability is surprisingly opened by production of these isotopes in subsequent $\alpha$-decays of elements 116 and/or 117 produced in the ${ }^{48} \mathrm{Ca}+{ }^{250} \mathrm{Cm}$ and ${ }^{48} \mathrm{Ca}+{ }^{249} \mathrm{Bk}$ fusion reactions, see Fig. 2. The corresponding cross sections of these reactions are not very low, they are about $0.8 \mathrm{pb}$ and $0.3 \mathrm{pb}$, correspondingly [1,2]. For the moment, this is the only method which is proposed for the production of SH nuclei located just in the middle of the island of stability.

\section{Neutron Capture Process}

The neutron capture process is an alternative (oldest and natural) method for the production of new heavy elements. The synthesis of heavier nuclei in the multiple neutron capture reactions with subsequent $\beta^{-}$decay is a well studied process.

The key quantity here is the time of neutron capture, $\tau_{n}=\left(n_{0} \sigma_{n \gamma}^{Z, A}\right)^{-1}$, where $n_{0}$ is the neutron flux (number of neutrons per square centimeter per second) and $\sigma_{n \gamma}^{Z, A}$ is the neutron capture cross section. If $\tau_{n}$ is shorter than the half-life of a given nucleus $T_{1 / 2}(Z, A)$ then the next nucleus $(Z, A+1)$ is formed by neutron capture. Otherwise the nucleus $(Z, A)$ decays before it captures next neutron. In nuclear reactors typical value of $\tau_{n} \sim 1$ year, and the nucleosynthesis occurs along the stability line by a sequence of neutron capture and $\beta^{-}$decay processes breaking at the short-living fissile fermium isotopes ${ }^{258-260} \mathrm{Fm}$ (so called "fermium gap"). In nuclear explosion $\tau_{n} \sim 1 \mu \mathrm{s}$, and more than 20 neutrons can be captured by a nucleus before it decays.

Strong neutron fluxes might be provided by nuclear reactors and nuclear explosions under laboratory conditions and by supernova explosions in nature. Theoretical models predict also another region of short-living nuclei located at $\mathrm{Z}=106 \div 108$ and $\mathrm{A} \sim 270$. In nuclear and supernova explosions (fast neutron capture) both these gaps may be bypassed if the total neutron fluence is high enough. Note that elements 99 and 100 (einsteinium and fermium) were first discovered in debris from the test thermonuclear explosion "Mike" [3].

The resulting charge number of the synthesized nuclei might be increased by sequential neutron flux exposure if 
two or several nuclear explosions were generated in close proximity to each other. The result depends both on the neutron fluence $n=n_{0} \tau_{\text {pulse }}$ and on the time interval between two exposures. The neutron fluence should be high enough to shift the produced neutron rich isotopes to the right from the second gap of unstable fissile nuclei located at $Z=106 \div 108$ and $A \sim 270$. Dependence on the time interval between two exposures is not so crucial if it is longer than several milliseconds (to avoid approaching the neutron drip line after several exposures) and shorter than a few minutes to avoid $\beta^{-}$decay of the produced nuclei into the area of fission instability.

Recently it was shown that the multiple rather "soft" nuclear explosions could be really used for the production of a noticeable (macroscopic) amount of neutron rich longliving SH nuclei [4]. We found a sharp increase of the probability for formation of heavy elements with $Z \geq 110$ in the multiple neutron irradiations: enhancement by several tens of orders of magnitude! The same process of multiple neutron exposures might be also realized in pulsed nuclear reactors of the next generation. An increase of the neutron fluence by about three orders of magnitude as compared with existing pulsed reactors could be quite sufficient to bypass both gaps [4].

\section{Multinucleon Transfer Reactions}

Renewed interest in the multinucleon transfer reactions with heavy ions is caused by the limitations of other reaction mechanisms for the production of new heavy and SH nuclei. The "northeast" area of the nuclear map cannot be reached in fusion, fission or fragmentation processes widely used nowadays for the production of new nuclei. Multinucleon transfer processes in near barrier collisions of heavy ions seem to be the most promising reaction mechanism allowing us to produce and explore neutronrich heavy nuclei including those located at the $\mathrm{SH}$ island of stability.

In our recent study we found that the shell effects may give us a gain in the yields of heavy neutron rich nuclei formed in multinucleon transfer reactions [1, 5-7]. In particular, the cross section for the production of unknown neutron rich nuclei located below ${ }^{208} \mathrm{~Pb}$ along the closed neutron shell $\mathrm{N}=126$ were predicted to be of several microbarns in low-energy collisions of ${ }^{136} \mathrm{Xe}$ or ${ }^{192} \mathrm{Os}$ with ${ }^{208} \mathrm{~Pb}$ target. Rather optimistic predictions were obtained also for the production of SH nuclei. For near barrier collisions of ${ }^{238} \mathrm{U}$ with ${ }^{248} \mathrm{Cm}$ cross sections higher than $1 \mathrm{pb}$ have been predicted for the production of new neutron enriched isotopes of elements with $\mathrm{Z} \leq 106$ located already at the stability line or even beyond it.

These are the shell effects which may significantly enhance the yield of $\mathrm{SH}$ nuclei for appropriate projectiletarget combinations. In Fig. 3 the charge and mass distributions of heavy primary reaction fragments are shown for near barrier collisions of ${ }^{48} \mathrm{Ca}$ and ${ }^{238} \mathrm{U}$ with curium target. The "lead peak" manifests itself in both reactions. However, for ${ }^{48} \mathrm{Ca}+{ }^{248} \mathrm{Cm}$ collisions it corresponds to the conventional (symmetrizing) quasi-fission process in which nucleons are transferred mainly from a heavy target (here it is ${ }^{248} \mathrm{Cm}$ ) to lighter projectile. This is a well studied process both experimentally and theoretically. It is caused just by the shell effects leading to the deep lead valley on the multi-dimensional potential energy surface which regulates the dynamics of the heavy nuclear system at low excitation energies.

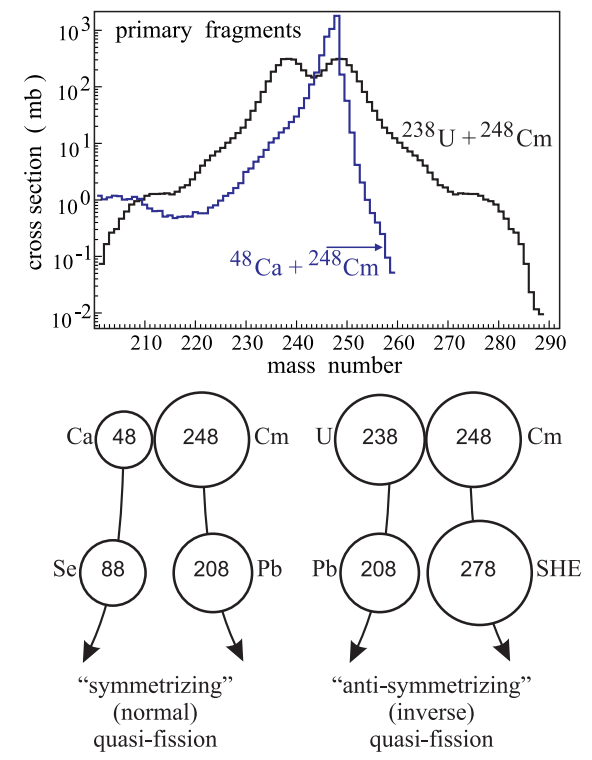

Figure 3. Calculated mass distributions of heavy primary reaction fragments formed in collisions of ${ }^{48} \mathrm{Ca}$ and ${ }^{238} \mathrm{U}$ with ${ }^{248} \mathrm{Cm}$ target at $E_{\text {c.m. }}=220$ and $770 \mathrm{MeV}$, correspondingly. Schematic view of conventional and "inverse" quasi-fission processes are also shown.

Contrary to this conventional quasi-fission phenomena, in low-energy collisions of ${ }^{238} \mathrm{U}$ with ${ }^{248} \mathrm{Cm}$ target nucleons may predominantly move from the lighter partner (here, uranium) to the heavy one, i.e., $\mathrm{U}$ transforms to a $\mathrm{Pb}$-like nucleus and $\mathrm{Cm}$ to the complementary $\mathrm{SH}$ nucleus. In this case, appearance of the lead shoulder in the mass and charge distributions of the reaction fragments automatically leads to a pronounced shoulder in the region of SH nuclei (see Fig. 3). We named it "inverse" (antisymmetrizing) quasi-fission process [5]. This process may really lead to enhanced yields of above-target nuclides, whereas even for rather heavy projectiles (like ${ }^{136} \mathrm{Xe}$ ) the nuclear system has a dominating symmetrizing trend of formation of reaction fragments with intermediate masses (heavier than projectile and lighter than target), see Fig. 4.

A possibility for the production of new heavy neutron rich nuclei in low-energy multinucleon transfer reactions is discussed currently in several laboratories. It is rather difficult to perform such experiments because of the low cross sections, the low intensities of these massive projectile beams and the problems of detecting the reaction products. In this connection, realistic predictions of the corresponding cross sections for different projectiletarget combinations are required as well as test experiments which may confirm or disprove these predictions. 


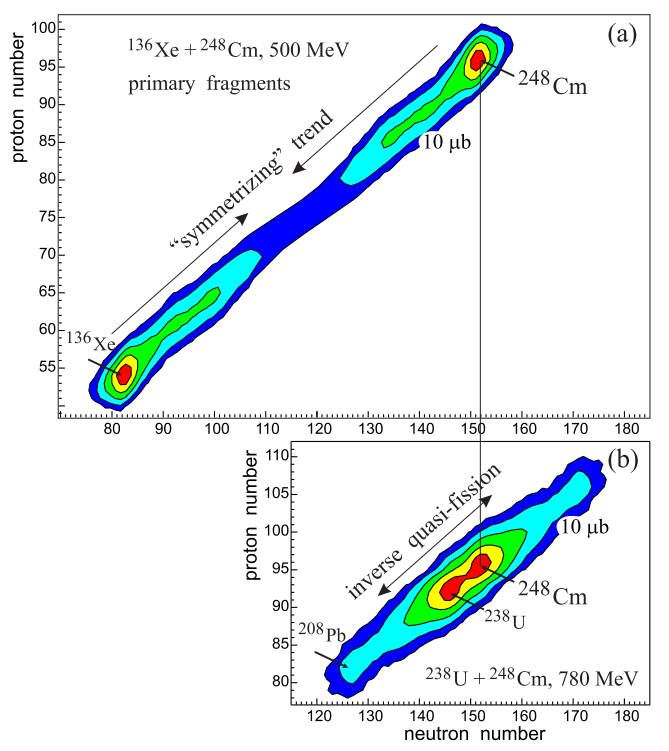

Figure 4. Landscapes of the calculated cross sections for the production of primary reaction fragments in collisions of ${ }^{136} \mathrm{Xe}$ (a) and ${ }^{238} \mathrm{U}$ (b) with ${ }^{248} \mathrm{Cm}$ target at $E_{\text {c.m. }}=500$ and $780 \mathrm{MeV}$, correspondingly (contour lines are drawn over one order of magnitude).

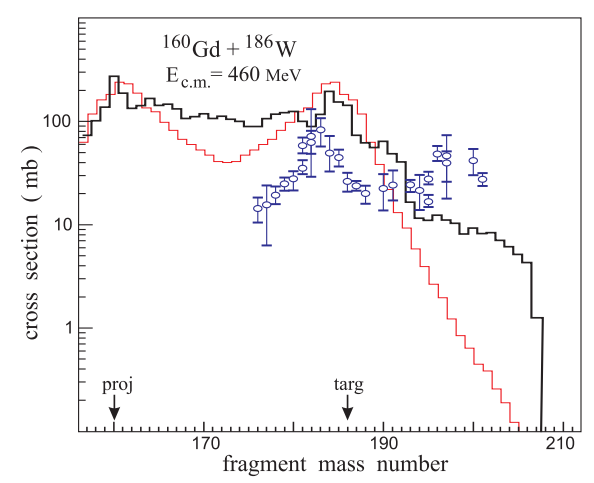

Figure 5. Predicted [8] and observed [9] mass distributions of reaction fragments (with energy loss higher than $15 \mathrm{MeV}$ ) formed in collisions of ${ }^{160} \mathrm{Gd}$ with ${ }^{186} \mathrm{~W}$ at $E_{\text {c.m. }}=460 \mathrm{MeV}$. Experimental data are shown only for target-like fragments. Thick and thin histograms show the results of calculations with and without (pure Liquid Drop Model) shell corrections in potential energy.

Some time ago a test (surrogate to $\mathrm{U}+\mathrm{Cm}$ ) reaction of ${ }_{64}^{160} \mathrm{Gd}_{\mathrm{N}=96}+{ }_{74}^{186} \mathrm{~W}_{\mathrm{N}=112}$ has been proposed [8], in which the same "inverse" quasi-fission process was expected due to neutron closed shells $\mathrm{N}=82$ and $\mathrm{N}=126$ located from the outside of the colliding partners. Recently such experiment has been performed [9]. Predicted and observed mass distributions of target-like nuclides formed in this reaction at $E_{\text {c.m. }}=460 \mathrm{MeV}$ are shown in Fig. 5.

Pronounced shoulder (as compared with conventional Liquid Drop Model for potential energy surface used for the calculation of nucleon transfer) has been predicted for the yields of trans-target nuclei (see the thick histogram in Fig. 5). Experimental data testify that the shell effects in low-energy multinucleon transfer reactions could be even stronger than it was expected. Cross sections for transfer of more than 15 nucleons from the lighter projectile to the heavier target were found to be higher than $20 \mathrm{mb}$. Unfortunately, experimental technique (catcher foils + offline radiochemistry) did not allow to measure the yields of stable (as well as short-living) isotopes and to reach the region of expected decrease of the cross sections for the production of trans-lead nuclei (see Fig. 5).

The calculated cross sections for the formation of heaviest trans-target nuclei in multinucleon transfer reactions underestimate the corresponding experimental data by about 1 order of magnitude. However available experimental data on the production of heavy trans-target nuclei in low-energy multinucleon transfer reactions are still insufficient and fragmentary. This impedes improving the model to make more accurate predictions (especially in the SH mass area). Experiments of such kind are very difficult to be performed due to the problems of on-line identification of heavy reaction products. Nevertheless additional experimental data in this field are urgently needed not only for better estimations of $\mathrm{SH}$ element production but also for better understanding of the mechanisms of multinucleon transfers in low-energy collisions of very heavy ions and for experimental determination of such fundamental quantities of nuclear dynamics as nucleon transfer rate and nuclear viscosity.

\subsection{The Model}

Several models have been proposed and used for the description of mass transfer in deep inelastic heavy ion collisions, namely, the Focker-Planck [10] and master equations [11] for the corresponding distribution function, the Langevin equations [12], and more sophisticated semiclassical approaches [13-15]. The well known GRAZING code [16] for description of nucleon transfer reactions in heavy ion collisions is also available on the market (recently it becomes possible to run this code directly at the NRV web-site [17]). The semiclassical model used by this code describes quite well few nucleon transfer reactions. However the multinucleon transfer processes cannot be described within this model, it gives too narrow mass distributions of reaction fragments because the damped reaction channels with large kinetic energy loss are not included in this model.

Calculations performed within the microscopic timedependent Schrödinger equations [18] have clearly demonstrated that at low collision energies of heavy ions nucleons do not "suddenly jump" from one nucleus to another. Instead of that, the wave functions of valence nucleons occupy the two-center molecular states spreading gradually over volumes of both nuclei (see Fig. 6).

The same adiabatic low-energy collision dynamics of heavy ions was found also within the TDHF calculations [19]. This means that the perturbation models based on a calculation of the sudden overlapping of single-particle wave functions of transferred nucleons (in donor and acceptor nuclei, respectively) cannot be used for description of multinucleon transfer and quasi-fission processes 


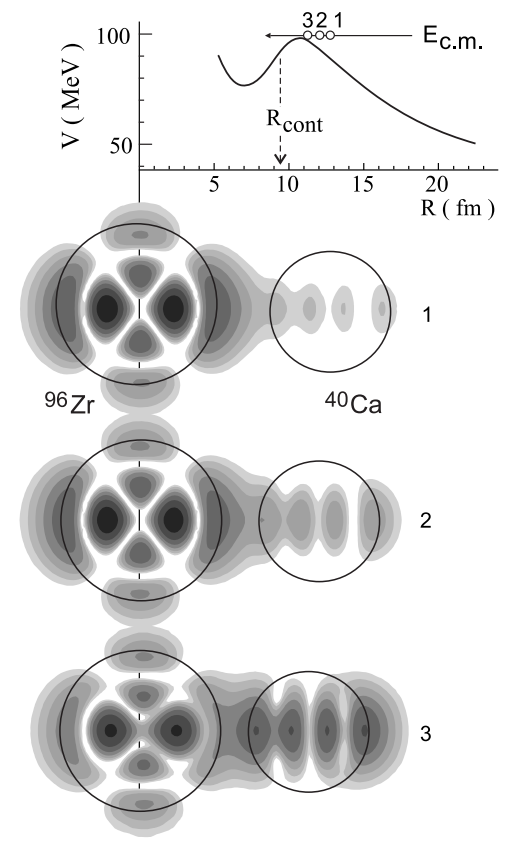

Figure 6. Amplitude of the wave function of valence neutron (initially located in the $2 d$ state of ${ }^{96} \mathrm{Zr}$ nucleus) at the three distances between colliding nuclei shown by the small circles in the upper part of the figure.

in low-energy heavy-ion damped collisions. Indeed the two center shell model and the adiabatic potential energy look most appropriate for the quantitative description of such processes.

The model based on the Langevin-type dynamical equations of motion was proposed recently $[20,21]$ for simultaneous description of strongly coupled multinucleon transfer, quasi-fission and fusion-fission reaction channels (difficult-to-distinguished experimentally in many cases). The distance between the nuclear centers $R$ (corresponding to the elongation of a mono-nucleus when it is formed), dynamic spheroidal-type surface deformations $\delta_{1}$ and $\delta_{2}$, the neutron and proton asymmetries, $\eta_{N}=$ $\left(2 N-N_{C N}\right) / N_{C N}, \eta_{Z}=\left(2 Z-Z_{C N}\right) / Z_{C N}$ (where $N$ and $Z$ are the neutron and proton numbers in one of the fragments, whereas $N_{C N}$ and $Z_{C N}$ refer to the whole nuclear system) are the most relevant degrees of freedom for the description of mass and charge transfer in deep inelastic scattering jointly with fusion-fission dynamics.

In low-energy damped collisions of heavy ions just the multi-dimensional potential energy surface regulates to a great extent the evolution of the nuclear system. In our approach we use a time-dependent potential energy, which after contact gradually transforms from a diabatic potential energy into an adiabatic one: $V\left(R, \delta_{1}, \delta_{2}, \eta_{N}, \eta_{Z} ; t\right)=$ $V_{\text {diab }}[1-f(t)]+V_{\text {adiab }} f(t)$ [20]. Here $t$ is the time of interaction and $f(t)$ is a smoothing function satisfying the conditions $f(t=0)=0$ and $f\left(t>>\tau_{\text {relax }}\right)=1, \tau_{\text {relax }}$ is an adjustable parameter $\sim 10^{-21} \mathrm{~s}$.

The diabatic potential energy is calculated within the double-folding procedure at the initial reaction stage, whereas in the adiabatic reaction stage we use the extended version of the two-center shell model [22], computational version of which can be found at the website [23]. Note that the diabatic, $V_{\text {diab }}$, and adiabatic, $V_{\text {adiab }}$, potential energies depend on the same variables and they are equal to each other for well separated nuclei. Thus, the total potential energy, $V\left(R, \delta_{1}, \delta_{2}, \eta_{N}, \eta_{Z} ; t\right)$, is a quite smooth function of all the parameters providing smooth driving forces, $-\partial V / \partial q_{i}$, at all reaction stages.

\subsection{Cross Sections}

The double differential cross-sections of all the binary reaction channels are calculated as follows

$$
\frac{d^{2} \sigma_{N, Z}}{d \Omega d E}(E, \theta)=\int_{0}^{\infty} b d b \frac{\Delta N_{N, Z}(b, E, \theta)}{N_{\mathrm{tot}}(b)} \frac{1}{\sin (\theta) \Delta \theta \Delta E} .
$$

Here $\Delta N_{N, Z}(b, E, \theta)$ is the number of events at a given impact parameter $b$ in which a nucleus $(N, Z)$ is formed in the exit channel with kinetic energy in the region $(E, E+\Delta E)$ and with center-of-mass outgoing angle in the interval $(\theta, \theta+\Delta \theta), N_{\text {tot }}(b)$ is the total number of simulated events for a given value of the impact parameter. This number depends strongly on low level of the cross section which one needs to be reached in calculation. For predictions of rare events with the cross sections of $1 \mu \mathrm{b}$ (primary fragments) one needs to test not less than $10^{7}$ collisions (as many as in real experiment).
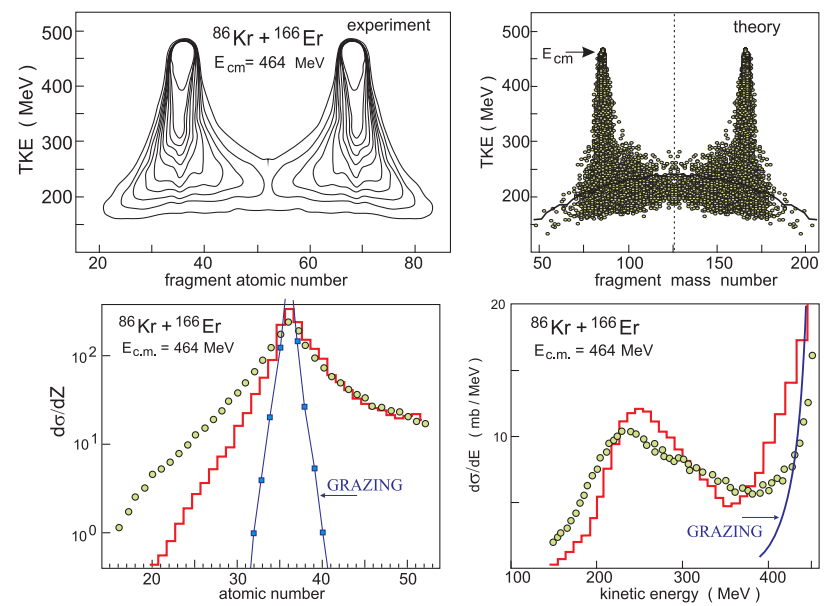

Figure 7. Charge, mass and energy distributions of reaction fragments in collisions of ${ }^{86} \mathrm{Kr}$ with ${ }^{166} \mathrm{Er}$ at $E_{\text {c.m. }}=464 \mathrm{MeV}$. On the two bottom panels the histograms indicate the calculations performed within the model described above whereas the curves show the GRAZING calculations.

Expression (1) describes the mass, charge, energy and angular distributions of the primary fragments formed in the binary reaction. Subsequent de-excitation cascades of these fragments via emission of light particles and gammarays in competition with fission are taken into account explicitly for each event within the statistical model leading to the final distributions of the reaction products. All the decay widths can be also calculated directly at the NRV website [24]. The sharing of the excitation energy between 

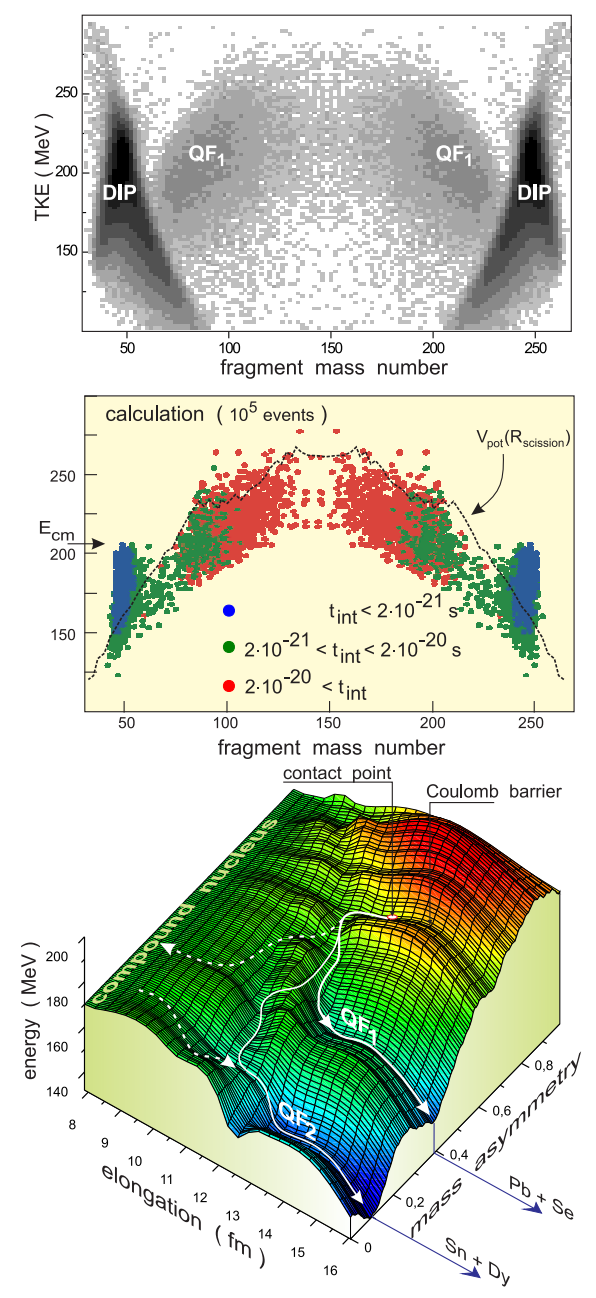

Figure 8. TKE-mass distribution of reaction fragments in collisions of ${ }^{48} \mathrm{Ca}$ with ${ }^{248} \mathrm{Cm}$ at $E_{\text {c.m. }}=203 \mathrm{MeV}$. All theoretical events are divided into 3 groups in accordance with reaction time. At the bottom panel adiabatic potential energy surface for this nuclear system is shown depending on distance between two centers and mass asymmetry (at fixed values of deformations of reaction fragments). The curves show schematically the trajectories leading to quasi-fission (solid curves) and fusion-fission (dashed) reaction channels.

the primary fragments is assumed here to be proportional to their masses (which is also debatable problem).

For the moment this approach is the only one which reproduces quite properly all the regularities of heavy ion deep inelastic scattering and quasi-fission processes $[20,21,25] . \quad$ As an example, in Fig. 7 experimental and theoretical energy-mass distributions of reaction fragments are shown formed in collisions of ${ }^{86} \mathrm{Kr}$ with ${ }^{166} \mathrm{Er}$ [26]. In Fig. 8 experimental [27] and theoretical energymass distributions are shown for quasi-fission reaction products in low-energy collisions of ${ }^{48} \mathrm{Ca}$ with ${ }^{248} \mathrm{Cm}$ at $E_{\text {c.m. }}=203 \mathrm{MeV}$. Potential energy surface for this nuclear system is also shown with schematic trajectories leading to different reaction channels. Theoretical events are divided into 3 groups in accordance with reaction time (difference between re-separation time of two fragments in the exit channel and contact time of colliding nuclei). As can be seen larger mass transfer really needs longer reaction time.

\subsection{Production of Transfermium Nuclei}

In Fig. 9 the results of our new calculations are shown for the formation of primary and survived isotopes of some transfermium elements in the reaction ${ }^{238} \mathrm{U}+{ }^{248} \mathrm{Cm}$ at the center-of-mass energy $770 \mathrm{MeV}$. The obtained results are rather optimistic. As can be seen, new neutron rich isotopes of transfermium elements with $Z=100-104$ (located already at the stability line and beyond it) can be produced with the cross sections of several hundreds of picobarn. The cross sections for the production of new neutron rich isotopes of seaborgim and hassium $(Z=106,108)$ are also higher than 1 picobarn. Note that the enhanced yield of primary trans-target nuclei with $A>265$ in this reaction is conditioned just by the shell effects (see also Fig. 3 ), namely, by decreasing of the potential energy in the channels with formation of projectile-like-fragments close to the doubly magic nucleus ${ }^{208} \mathrm{~Pb}$.

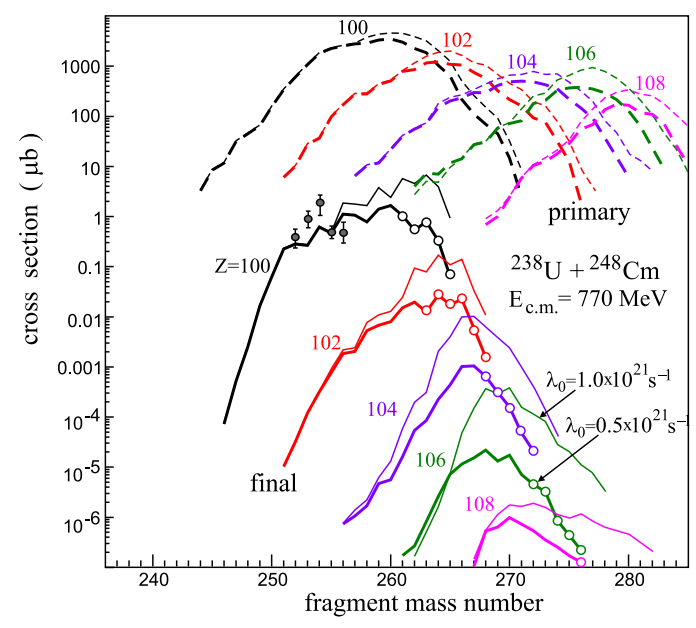

Figure 9. Cross sections for the production of transfermium nuclei in collisions of ${ }^{238} \mathrm{U}$ with ${ }^{248} \mathrm{Cm}$ target at $E_{\text {c.m. }}=770 \mathrm{MeV}$ calculated at two values of the nucleon transfer rate: $\lambda_{0}=$ $0.5 \cdot 10^{21} \mathrm{~s}^{-1}$ (thick curves) and $\lambda_{0}=1.0 \cdot 10^{21} \mathrm{~s}^{-1}$ (thin curves). Open circles indicate new isotopes of transfermium elements (numbers near the curves). The dashed curves indicate the cross sections for the production of primary fragments. Experimental data are taken from [28] for the production of fermium isotopes in this reaction at beam energy $E_{\text {c.m. }}=862 \mathrm{MeV}$ before entering the thick ${ }^{248} \mathrm{Cm}$ target.

Owing to extremely low survival probability of excited heavy primary trans-target reaction fragments the cross sections for the production of SH nuclei located just in the middle of the island of stability $(Z \sim 112, N \sim 180)$ were found to be less than $1 \mathrm{pb}$ in this reaction $[1,7]$. This is too low to perform experiments aimed on the production of these nuclei at available facilities. However, as mentioned above, our model probably underestimates the yields of primary trans-target reaction fragments.

A rather unusual dependence of the calculated cross sections on the value of the nucleon transfer rate $\lambda_{0}$ was 
found. Increasing this parameter by a factor of 2 leads to an increase (also by a factor of 2 or 3 ) only in the yields of neutron-enriched primary trans-target fragments (see thin dashed curves in Fig. 9). At the same time, this leads to an increase in the yields of final (surviving) neutron-enriched isotopes of trans-target elements by more than one order of magnitude. This means that larger values of the nucleon transfer rate $\lambda_{0}$ may lead in this reaction to formation of less excited heavy trans-target nuclei at earlier reaction stage (lower excitation energy means higher survival probability and smaller number of evaporated neutrons). Additional uncertainty of the predictions for the formation of neutron enriched transfermium nuclei in multinucleon transfer reactions arises from the uncertainty of their survival probability, which strongly depends on the fission barriers of these nuclei, which also cannot be estimated very accurately. Taken together these factors mean that at the moment one cannot make such predictions very precisely, so at least factor of 10 should be kept in mind.

\subsection{Downward along $\mathrm{N}=126$}

Actinide beams (as well as actinide targets) might be successfully used also for the production of new neutron rich nuclei around the closed neutron shell $N=126$, the region having largest impact on astrophysical $r$ process. Near barrier collisions of ${ }^{136} \mathrm{Xe}$ and ${ }^{192} \mathrm{Os}$ with ${ }^{208} \mathrm{~Pb}$ target were predicted to be quite promising for the production of new nuclei with $N \sim 126[6,7]$. The corresponding cross sections were found to be about $1 \mu \mathrm{b}$ and less. The use of heavy radioactive ion beams ( such as ${ }^{132} \mathrm{Sn}$ or ${ }^{154} \mathrm{Xe}$ ) gives a gain in the nucleon transfer cross sections but not in the final yields of new neutron rich nuclei because of low intensity of these beams.

Low-energy collisions of stable neutron enriched isotopes of elements located below lead (such as ${ }^{192}$ Os or ${ }^{198} \mathrm{Pt}$ ) with available actinide nuclei look even more favorable for the production and study of new neutron rich nuclei located around neutron closed shell $N=126$. Distribution of primary fragments formed in transfer reactions are concentrated around the line connecting projectile and target (just due to conservation of proton and neutron numbers). If one of reaction partner has a neutron excess (like ${ }^{238} \mathrm{U}$ ), then this line will be inclined to neutron axis. Distribution of primary fragments in $(Z, N)$ plane is shown in Fig. 10 for the case of transfer reaction products formed in low energy collisions of ${ }^{198} \mathrm{Pt}$ with ${ }^{238} \mathrm{U}$ at $E_{\mathrm{c} . \mathrm{m} .}=700 \mathrm{MeV}$. As can be seen a lot of new isotopes in the region of the closed neutron shell $N=126$ can be synthesized in this reaction.

Estimated cross sections for the production of the final (survived) isotopes of the elements with $Z=71 \div 78$ in low energy collisions of ${ }^{198} \mathrm{Pt}$ with ${ }^{238} \mathrm{U}$ are shown in Fig. 11. On average, the cross sections for the production of new neutron rich heavy nuclei (including those located along the closed neutron shell $N=126$ ) in this reaction are higher than in collisions of ${ }^{136} \mathrm{Xe}$ or ${ }^{192} \mathrm{Os}$ with ${ }^{208} \mathrm{~Pb}$ target [7] (though a contamination by uranium fission fragments probably may reduce this gain in the cross sections).

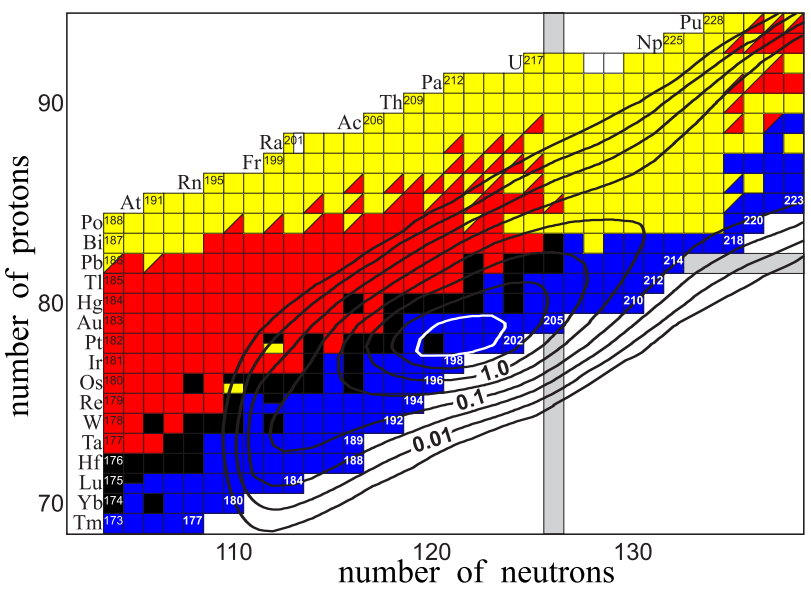

Figure 10. Contour plot of the cross sections (in logarithmic scale) for the formation of primary reaction fragments in collisions of ${ }^{198} \mathrm{Pt}$ with ${ }^{238} \mathrm{U}$ at $E_{\text {c.m. }}=700 \mathrm{MeV}$. Contour lines are drawn over half order of magnitude and units of measurement are millibarns (numbers on lines).

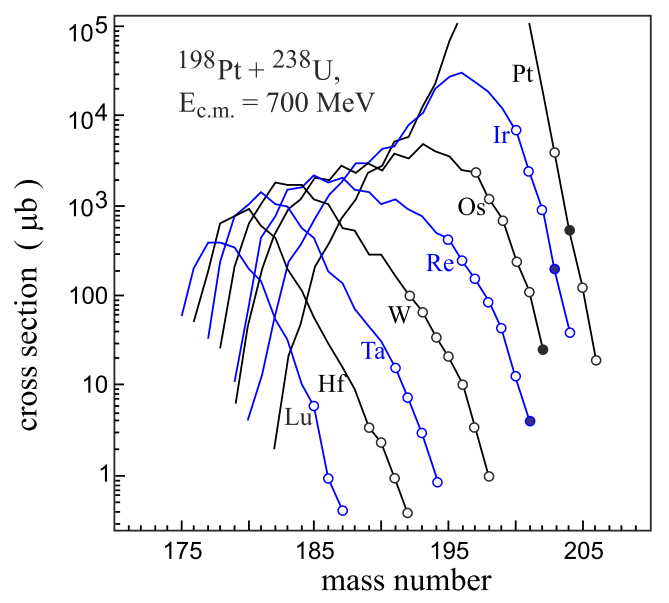

Figure 11. Isotopic yields of elements below lead (from Lu to Pt) in collisions of ${ }^{198} \mathrm{Pt}$ with ${ }^{238} \mathrm{U}$ at $E_{\text {c.m. }}=700 \mathrm{MeV}$. Circles denote not-yet-known isotopes (the solid ones show isotopes with closed neutron shell $N=126$ ).

\subsection{Project GaLS}

In contrast with fusion reactions it is more difficult to separate a given nucleus from all the transfer reaction products. The neutron rich heavy nuclei with $Z>70$ formed in the multinucleon transfer reactions cannot be separated and studied at available setups created quite recently just for studying the products of deep inelastic scattering (such as VAMOS, PRISMA and others). These fragment separators (as well as other setups) cannot distinguish heavy nuclei with $Z>70$ by their atomic numbers. However during the last several years a combined method of separation is intensively studied based on stopping nuclei in gas and subsequent resonance laser ionization of them. One of such setups (named GaLS: in gas cell laser ionization and separation) is currently created at Flerov Laboratory (JINR, Dubna). Experiments at this setup aimed on pro- 


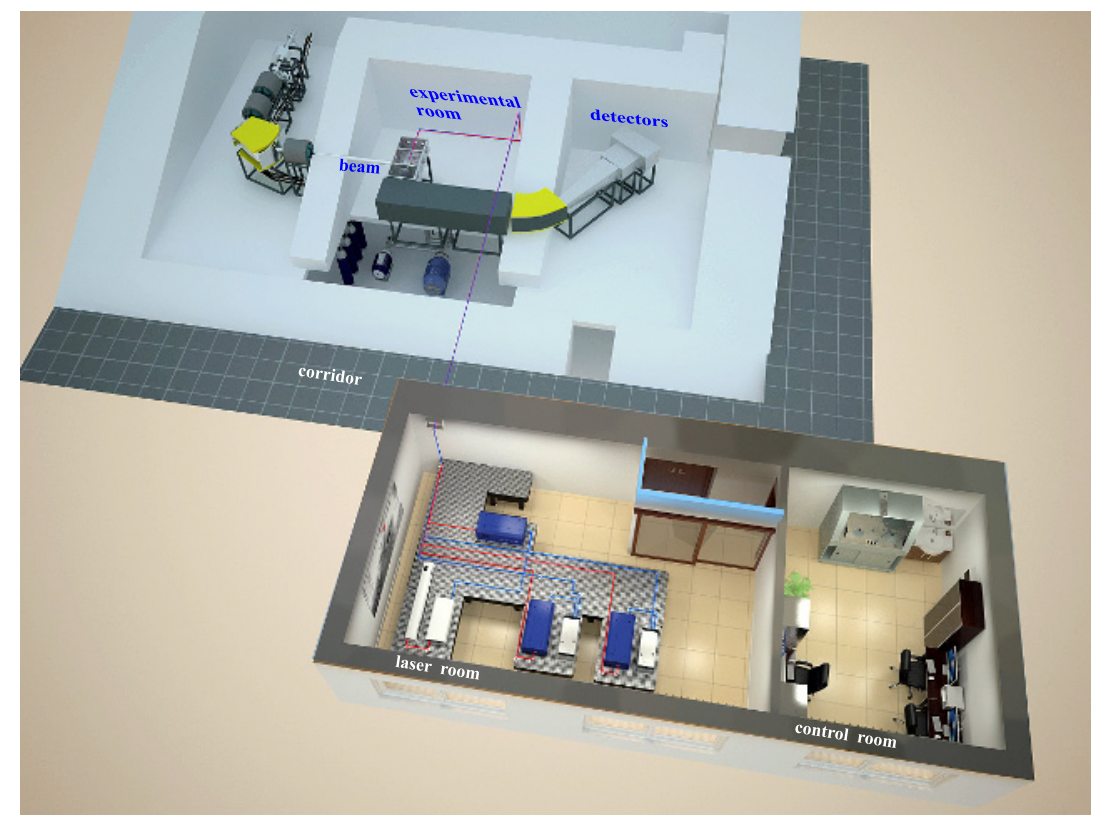

Figure 12. 3d view of the setup GaLS (Dubna) for selective laser ionization and separation of heavy transfer reaction products.

duction and studying properties of new neutron rich heavy nuclei are planned to start in 2015 (see Fig. 12).

\section{References}

[1] V.I. Zagrebaev and W. Greiner, Phys. Rev. C 78, 034610 (2008).

[2] V.I. Zagrebaev, A.V. Karpov and W. Greiner, Phys. Rev. C 85, 014608 (2012).

[3] H. Diamond, P.R. Fields, C.S. Stevens et al., Phys. Rev. 119, 2000 (1960).

[4] V.I. Zagrebaev, A.V. Karpov, I.N. Mishustin and W. Greiner, Phys. Rev. C 84, 044617 (2011).

[5] V.I. Zagrebaev, Yu.Ts. Oganessian, M.G. Itkis and W. Greiner, Phys. Rev. C 73, 031602 (2006).

[6] V. Zagrebaev and W. Greiner, Phys. Rev. Lett. 101, 122701 (2008).

[7] V.I. Zagrebaev and W. Greiner, Phys. Rev. C 83, 044618 (2011).

[8] V. Zagrebaev and W. Greiner, J. Phys. G 34, 2265 (2007).

[9] W. Loveland et al., Phys. Rev. C 83, 044610 (2011).

[10] W. Nörenberg, Phys. Lett. B 52, 289 (1974)

[11] L.G. Moretto and J.S. Sventek, Phys. Lett. B 58, 26 (1975).

[12] P. Fröbrich and S.Y. Xu, Nucl. Phys. A477, 143 (1988).

[13] E. Vigezzi and A. Winther, Ann. Phys. (N.Y.) 192, 432 (1989).
[14] V.I. Zagrebaev, Ann. Phys. (N.Y.) 197, 33 (1990).

[15] A. Winther, Nucl. Phys. A594, 203 (1995).

[16] http://personalpages.to.infn.it/nanni/grazing/.

[17] http://nrv.jinr.ru/nrv/webnrv/grazing/.

[18] V.I. Zagrebaev, V.V. Samarin and W. Greiner, Phys. Rev. C 75, 035809 (2007).

[19] C. Simenel, A. Wakhle and B. Avez, J. Phys.: Conf. Ser. 20, 012118 (2013).

[20] V. Zagrebaev and W. Greiner, J. Phys. G 31, 825 (2005).

[21] V. Zagrebaev and W. Greiner, J. Phys. G 34, 1 (2007).

[22] V. Zagrebaev, A. Karpov, Y. Aritomo, M. Naumenko, and W. Greiner, Phys. Part. Nucl. 38,469 (2007).

[23] V. Zagrebaev, A. Alekseev and A. Karpov, Two Center Shell Model code of NRV, http://ntr.jinr.ru/nrv/.

[24] V. Zagrebaev, A. Alekseev and A. Karpov, Statistical Model code of NRV, http://ntr.jinr.ru/nrv/.

[25] Y. Aritomo, Phys. Rev. C 80, 064604 (2009).

[26] A. Gobbi, U. Lynen, A. Olmi, G. Rudolf, and H. Sann, in Proc. Int. School of Phys. "Enrico Fermi", Course LXXVII, Varenna, 1979 (North-Holland, Amsterdam, 1981) 1.

[27] M.G. Itkis et al., Nucl. Phys. A734, 136 (2004).

[28] M. Schädel, W. Brüchle, H. Gäggeler, J.V. Kratz, K. Sümmerer, G. Wirth, G. Herrmann, R. Stakemann, G. Tittel, N. Trautmann, J.M. Nitschke, E.K. Hulet, R.W. Lougheed, R.L. Hahn, and R.L. Ferguson, Phys. Rev. Lett. 48, 852 (1982). 